\title{
SDCEP Dental Prescribing app
}

\author{
Derek Richards \\ Editor
}

A new mobile app to facilitate drug prescribing in primary care has been launched by the Scottish Dental Clinical Effectiveness Programme (SDCEP). Dental Prescribing is available to purchase from the App Store ${ }^{\mathrm{SM}}$ for use on iPhone ${ }^{\circledR}, \mathrm{iPad}^{\circledR}$ and iPod touch ${ }^{\circledR}$.

The app is one of several guidance publications from SDCEP, a National Dental Advisory Committee (NDAC) initiative supported by NHS Education for Scotland (NES). Established in 2004, SDCEP aims to support improvements in the quality of dental care by providing user-friendly, evidence-based guidance on specific dental topics that are priorities in Scotland but which are also likely to be of relevance further afield.

SDCEP first published guidance on drug prescribing in primary care dental practice, entitled Drug Prescribing For Dentistry, in April 2008. With numerous changes in prescribing information since then, an updated second edition of the guidance was released in August 2011.

These spiral-bound A5 booklets (Fig. 1) contain advice specific to dental prescribing from the British National Formulary (BNF) and the BNF for Children (BNFC), divided according to the conditions that patients might present with in primary care dental practice. Prescribing information, with dose regimens, is provided for
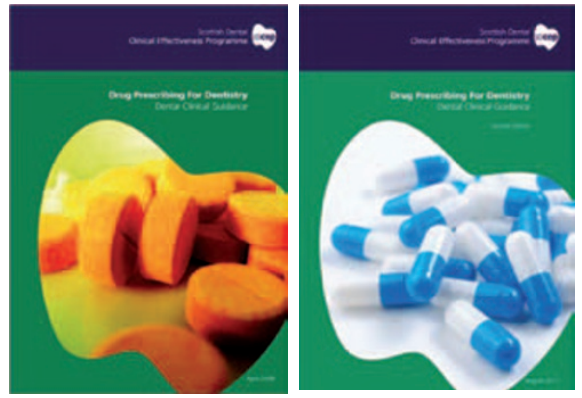

Fig. 12008 and 2011 Covers of Drug Prescribing For Dentistry guidance booklets

adults in a 'prescription-like' format and variations in doses and formulations used for different age ranges of children are provided alongside. Brief advisory notes and cautions are provided in footnotes to the prescribing boxes to help inform the decision of the practitioner. The guidance also includes advice on the management of medical emergencies, with a list of the drugs used to deal with such incidents, compatible with recommendations of the Resuscitation Council (UK) and the BNF. The SDCEP prescribing guidance states that it is intended to be used in conjunction with the BNF and BNFC, particularly for information on drug interactions, full details of contraindications, cautions and side effects, and prescribing for specific patient groups (e.g. renal

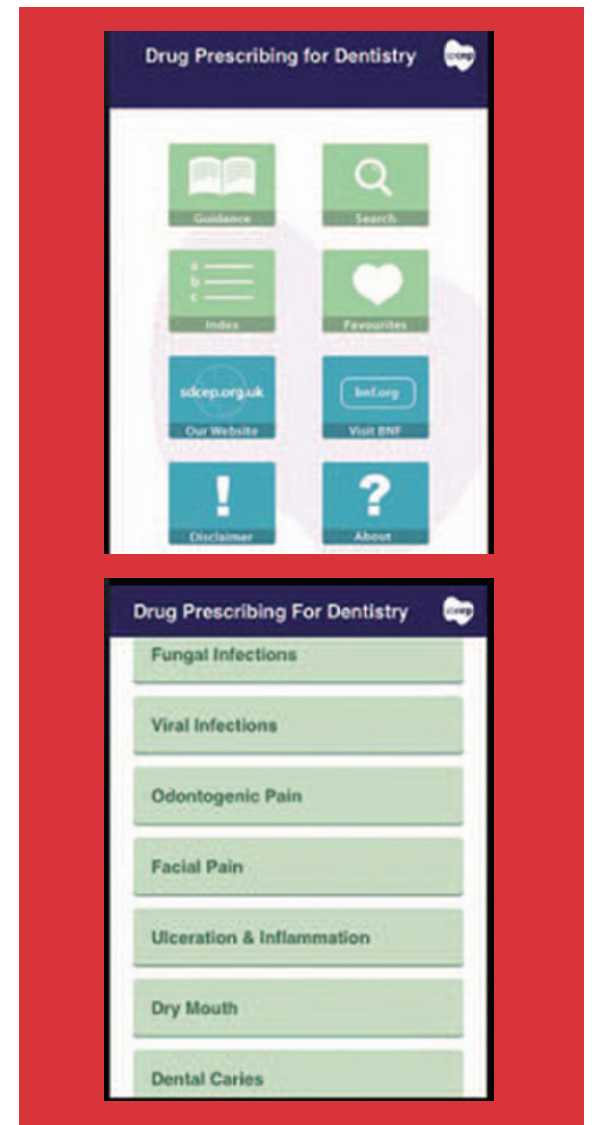

Fig. 2 Main category index

impairment, pregnancy). SDCEP has provided updates to the guidance to coincide with significant changes to dental prescribing advice within the BNF and BNFC.

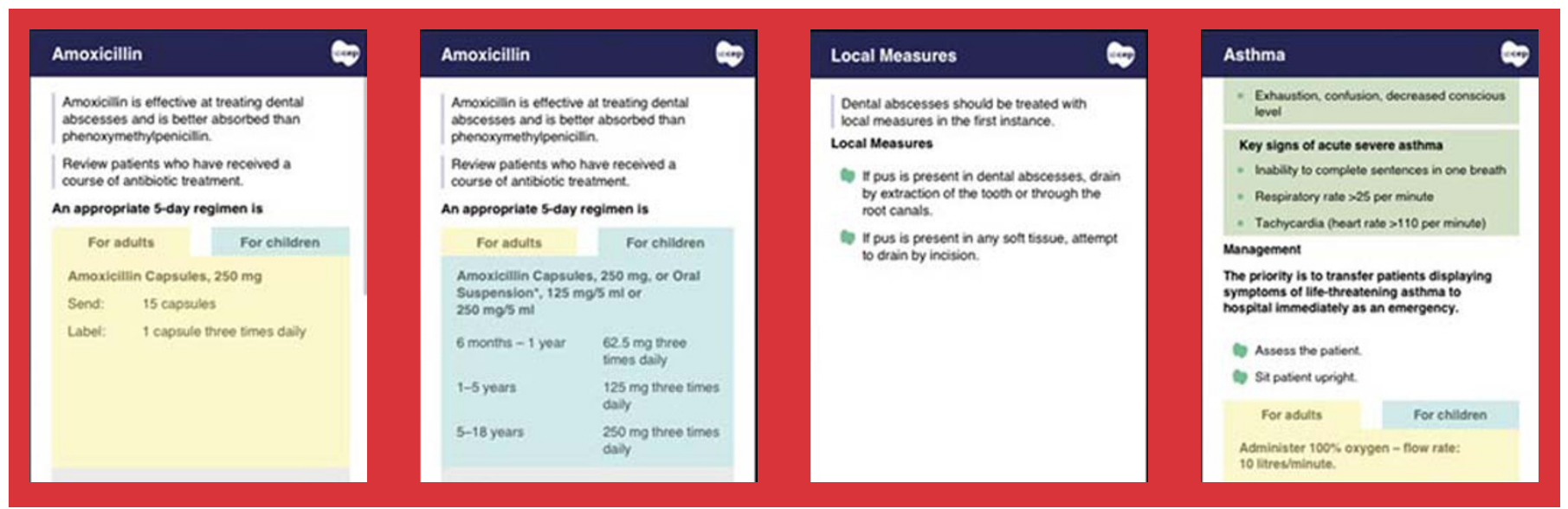

Fig. 3 Adult and child tabs, local measures and medical emergency pages 


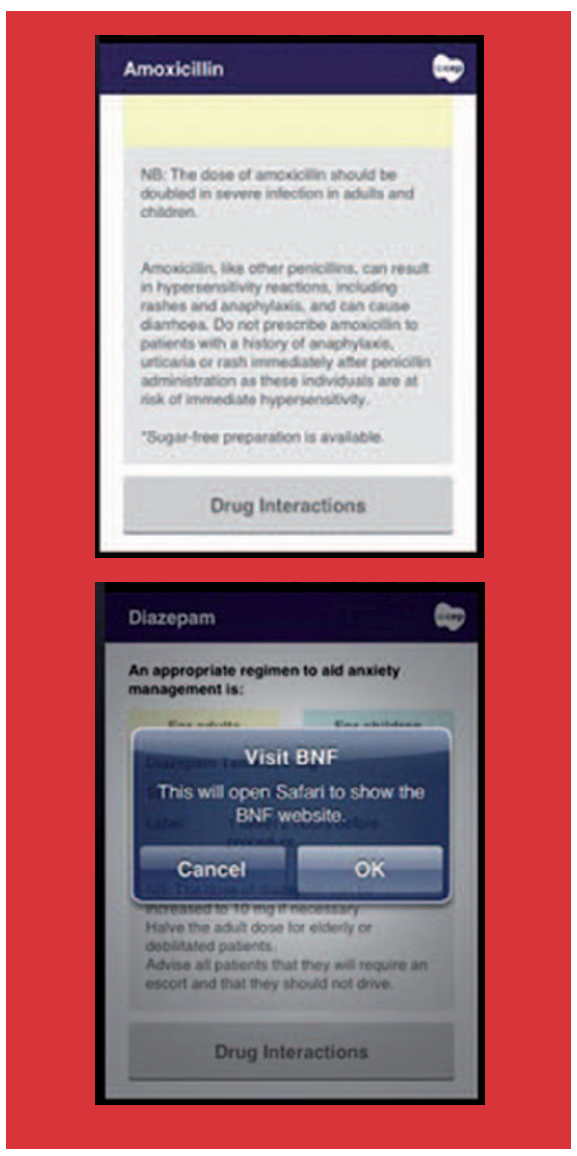

Fig. 4 Drug cautions and links to BNF

The new Dental Prescribing app is structured in the same way as the printed guidance, with advice on managing patients classified by condition in nine broad categories, including bacterial infections, odontogenic pain, ulceration \& inflammation and dental caries I (Fig. 2). Dose information for both adults and children is provided using a neat tab arrangement. Local measures to be used in the first instance or as an adjunct to prescribing are highlighted. Advice on the management of medical emergencies is also included (Fig. 3).

The app has been designed to be a quick reference tool and accordingly the advice accompanying the dose regimens has been condensed from the printed version to essential bullet points. However the full guidance text is available to view in Further Information screens. This feature is likely to be particularly useful for students and the less-experienced practitioner. The brief advisory notes and cautions from the print- ed guidance are retained. One of the app's most appealing features is the inclusion of numerous direct links to the BNF's website for drug interaction information. While this does require a web connection and registration with the BNF (free for UK users) this appears to be one of the big advantages of this format (Fig. 4).

The app includes other additional features to assist the busy practitioner. The Search function allows the user to quickly find advice on a drug or on the management of a specific dental condition, including local measures, while the Favourites function allows the user to bookmark frequently viewed screens for quick access. The user can also adjust the text size (Fig. 5). SDCEP intends to keep the app up to date with changes to the dental prescribing guidance from the BNF and BNFC, with free updates provided for at least two years following launch.

The app is likely to prove a useful tool for practitioners working in primary care and has received positive feedback on the app store, with five 5-star ratings to date, plus an enthusiastic review from a user. Undergraduate students and vocational trainees will find the app a handy study aid and more experienced practitioners will find it useful when reviewing notes and as a means of keeping up to date with the latest prescribing advice. The portability of the app will benefit those who work in multiple locations or those whose duties include domiciliary visits.

Details of this and other publications from SDCEP are provided on its website (www.sdcep.org.uk). These include guidance on conscious sedation, decontaminating instruments, emergency care, caries in children, oral health assessment and review and management of patients taking bisphosphonates. SDCEP also maintains the 'Practice Support Manual', an online resource which provides support for practice management and organisation in primary dental care. Future SDCEP guidance publications will cover the management of acute dental problems and periodontal care.

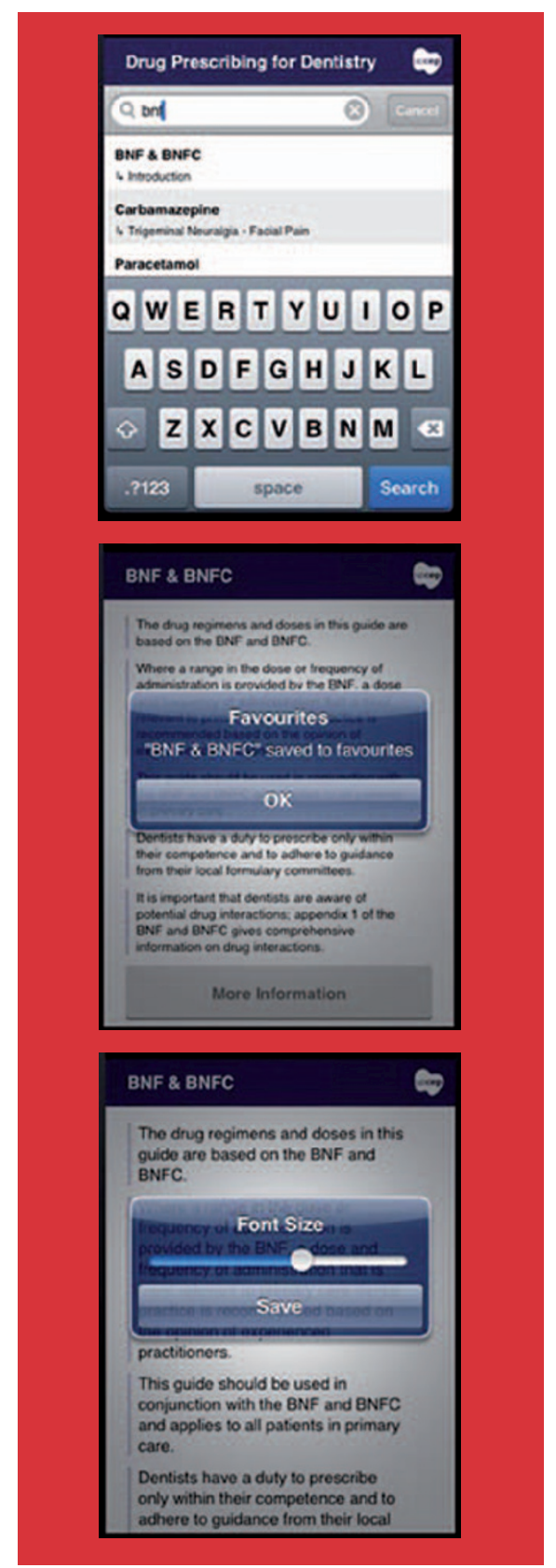

Fig. 5 Search, favourites and text size screens

Conflict of interest: Derek Richards works as a specialist advisor to the SDCEP Programme. However, he has not been directly involved with the development of the Drug Prescribing For Dentistry guidance or this app.

* Apple, the Apple logo, iPad, iPhone and iPod touch are trademarks of Apple Inc., registered in the U.S. and other countries. App Store is a service mark of Apple Inc.

Evidence-Based Dentistry (2012) 13, 61-62. doi:10.1038/sj.ebd.6400867. 